\title{
Uso de catéteres venosos periféricos y prevalencia de flebitis en un hospital de cuidados secundarios de La Habana
}

\section{Short peripheral venous catheter use and phlebitis incidence in a secondary care hospital of La Habana}

\author{
Anaika González-Valdés ${ }^{1}$, Teresa Cuní-Rivera², Dailín Santana-Ortíz², Luis González-Álvarez ${ }^{1}$ y \\ Humberto Guanche-Garcell ${ }^{1 *}$ \\ ${ }^{1}$ Departamento de Epidemiología; ²Departamento de Enfermería. Hospital Joaquín Albarrán, La Habana, Cuba
}

\begin{abstract}
Resumen
Objetivo: Describir la prevalencia de flebitis. Se realizaron estudios de prevalencia puntual semanalmente en 14 salas de pacientes hospitalizados en un hospital de atención secundaria de La Habana, Cuba. Material y método: Desde el primero de noviembre de 2018 hasta el 31 de enero de 2019, se llevaron a cabo 11 prevalencias puntuales, se evaluaron 2,282 pacientes y 1,087 pacientes tenían catéteres venosos periféricos (CVP) (razón 0.48). Resultados: Se identificó evidencia de flebitis en 63 pacientes (5.8\%). El uso de CVP fue más intenso en salas clínicas (razón 0.59) en comparación con salas de cuidados críticos (razón 0.45) $(p=0.00)$ y salas quirúrgicas $(0.35)(p=0.00)$. La prevalencia de flebitis fue del 3.2, 5.6 y $6.5 \%$ en salas de cirugía, cuidados críticos y clínicas, respectivamente. El enrojecimiento (100\%), edema (84.5\%) y dolor (60\%) fueron los signos más frecuentes de flebitis. Conclusión: Este estudio proporciona información sobre el uso de CVP y la flebitis, que debe ser utilizada por el programa local de mejora de la calidad.
\end{abstract}

Palabras clave: Catéter venoso periférico corto. Razón de utilización. Flebitis. Prevalencia. Cuba.

\section{Abstract}

Objective: Describe the prevalence of phlebitis in admitted patients Methods: Its were conducted point prevalence studies on weekly basis in 14 inpatients wards in a secondary care hospital (La Habana, Cuba). From November $1^{\text {st }}, 2018$ to January 31, 2019, 11 point prevalence was carried out, 2282 patients were evaluated and 1087 patients had SPC (ratio 0.48). Results: Evidence of phlebitis was identified in 63 patients (5.8\%). SPC use was more intense in clinical wards (ratio 0.59) compared with critical care wards (ratio 0.45) $(p=0.00)$ and surgical wards $(0.35)(p=0.00)$. Phlebitis incidence was $3.2 \%$, $5.6 \%$ and $6.5 \%$ in surgical, critical care and clinical wards respectively. Redness (100\%), edema (84.5\%) and pain (60\%) were the most frequent sign of phlebitis. Conclusion: This study provides insight into the SPC use and phlebitis that should be used by the local quality improvement program.

Key words: Short peripheral venous catheter. Utilization ratio. Phlebitis. Incidence. Cuba.

\section{Correspondencia:}

*Humberto Guanche-Garcell

E-mail: guanche@infomed.sld.cu
Disponible en internet: 19-06-2020 Rev Hosp Jua Mex. 2020;87(2):70-73

www.revistahospitaljuarez.com 1405-9622/○ 2020 Sociedad Médico-Quirúrgica del Hospital Juárez de México, A.C. Publicado por Permanyer. Este es un artículo open access bajo la licencia CC BY-NC-ND (http://creativecommons.org/licenses/by-nc-nd/4.0/). 


\section{Introducción}

Los catéteres venosos periféricos (CVP) son los dispositivos médicos invasivos más utilizados en los hospitales de todo el mundo y están relacionados con varios efectos adversos, como flebitis e infecciones relacionadas con el acceso vascular ${ }^{1,2}$. La flebitis es la complicación más frecuente de los catéteres periféricos, y se ha reportado en hasta el $80 \%$ de los pacientes con CVP, la cual se puede manifestar hasta 48 horas después de la extracción de los catéteres ${ }^{2,3}$.

Varios factores podrían estar relacionados con la flebitis, especialmente los factores de los pacientes (edad, sexo, comorbilidades), el catéter (tipo y tamaño del catéter, tiempo de exposición), la calidad de inserción y mantenimiento de los catéteres y las sustancias infundidas ${ }^{2-6}$.

En los centros de salud cubanos, no hay personal de enfermería dedicado para insertar CVP. La desinfección generalmente se realiza con alcohol etílico $(70 \% \mathrm{v} / \mathrm{v})$, y el uso de apósitos no semitransparentes es una práctica estándar. Además, el personal utiliza catéteres cortos en la mayoría de los pacientes que requieren acceso vascular, y la práctica de utilizar catéteres centrales insertados periféricos es poco común. La evidencia obtenida de la observación durante rondas regulares destaca la frecuencia de flebitis en pacientes ingresados y la posibilidad de uso excesivo de CVP.

El objetivo del estudio fue describir la prevalencia de flebitis en salas de hospitalización de un hospital de atención secundaria, como un paso inicial para llevar a cabo una intervención de mejora de la calidad.

\section{Métodos}

Se realizaron estudios de prevalencia puntual semanalmente en 14 salas de hospitalización (clínicas, quirúrgicas, de cuidados críticos) del Hospital Joaquín Albarrán, un hospital universitario de atención secundaria de 300 camas de La Habana, Cuba. El estudio se realizó entre noviembre de 2018 y enero de 2019.

Antes del inicio del estudio, dos licenciadas en enfermería con más de 15 años de experiencia fueron capacitadas en los procedimientos de recolección de datos. Durante un día, todos los pacientes ingresados fueron evaluados y registrados. Se documentó el número de pacientes ingresados por sala, el uso de CVP y la identificación de evidencias de flebitis (enrojecimiento, dolor, edema, cordón o vena palpable y flujo purulento).
Se calculó el índice de utilización de CVP (número de CVP colocados dividido por el número de pacientes ingresados) y la prevalencia de flebitis (por 100 pacientes con CVP).

En referencia a la aprobación ética, no se recopiló ningún identificador del paciente durante el estudio y los procedimientos de recopilación de datos siguieron la política y los procedimientos de infecciones asociadas a la asistencia sanitaria del centro. El consejo científico proporcionó una exención para el consentimiento informado y aprobó la publicación del estudio.

\section{Resultados}

Desde el primero de noviembre de 2018 hasta el 31 de enero de 2019, se realizaron 11 prevalencias puntuales para identificar la frecuencia del uso de CVP y la prevalencia de flebitis. Se evaluaron 2,282 pacientes, de los cuales, 1,087 pacientes tenían colocado un CVP (razón 0.48), y se identificaron evidencias de flebitis en 63 pacientes (5.8\%) (Tabla 1).

El uso de CVP fue más intenso en salas clínicas (razón 0.59) en comparación con salas de cuidados críticos (razón 0.45) $(p=0.00)$ y salas quirúrgicas (razón 0.35) $(p=0.00)$. Se observaron seis salas (42.9\%) con una razón de uso de CVP más elevada que la media del hospital, con la cifra más alta en la sala número nueve (razón 0.68), que se dedica a la atención de pacientes clínicos con predominio de infecciones adquiridas en la comunidad (neumonía e infecciones del tracto urinario). En esta sala se observó el índice de flebitis más alto (12.5\%). En otras cuatro salas, la prevalencia de flebitis fue superior a la media del hospital. En general, las salas con relación entre CVP y flebitis están dedicadas a atender pacientes clínicos. La prevalencia de flebitis fue del 3.2, 5.6 y $6.5 \%$ en salas de cirugía, cuidados críticos y clínica, respectivamente $(p=0.27)$ (Tabla 1 y Fig. 1).

En todos los pacientes con flebitis se observó enrojecimiento local, con orden descendente de frecuencia para el dolor $(60.0 \%)$, edema (84.5\%) y un cordón palpable en el $10 \%$ de los pacientes. No se observó pacientes con drenaje purulento proveniente del sitio de flebitis.

\section{Discusión}

Este estudio ha mostrado una baja prevalencia de flebitis y el uso de CVP, con frecuencias más altas en pacientes clínicos en comparación con pacientes quirúrgicos o críticos. Además, los resultados destacan la 


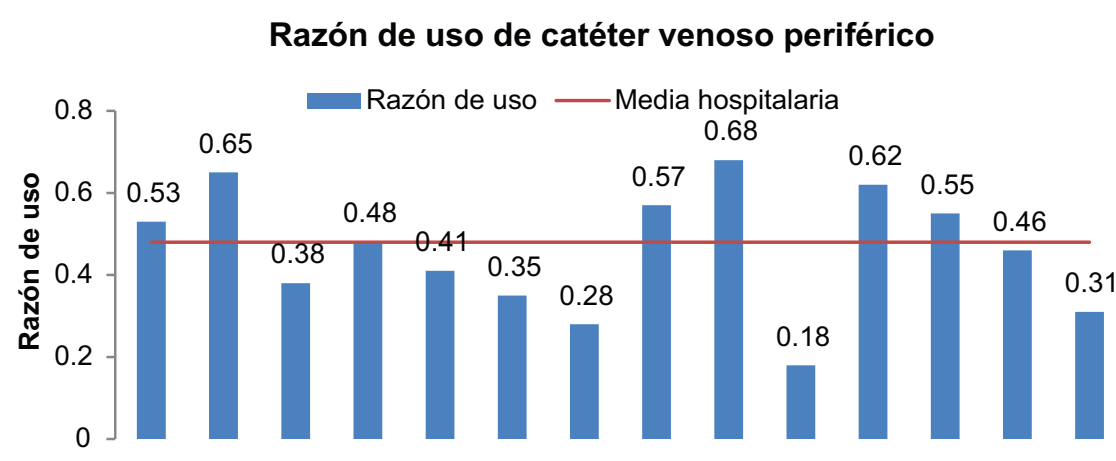

\section{Incidencia de flebitis}

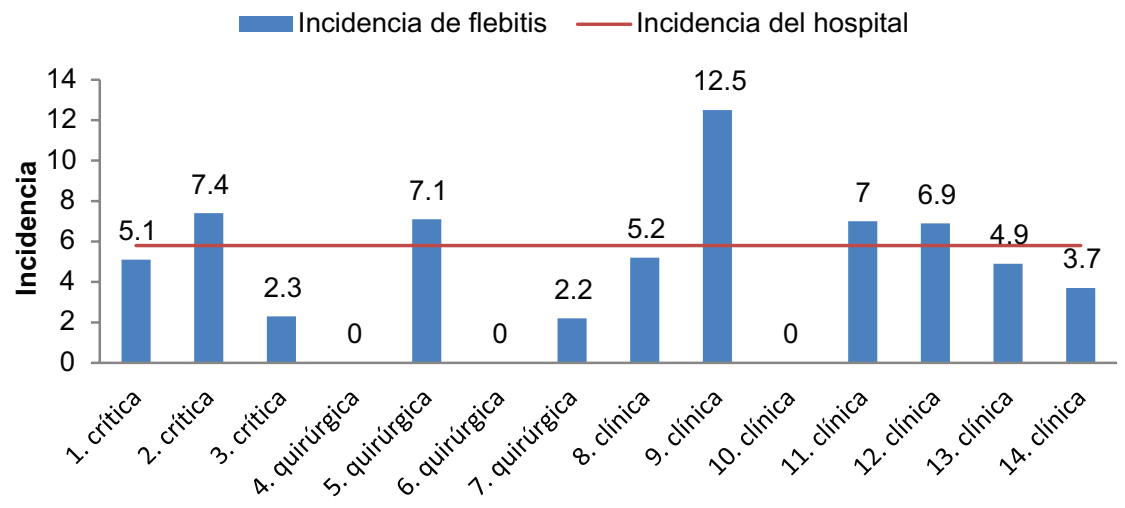

Figura 1. Razón de uso de catéter venoso periférico y prevalencia de flebitis (por 100 pacientes con catéteres venosos periféricos) de acuerdo con el perfil de las salas.

Tabla 1. Uso de catéter venoso periférico y evidencias de flebitis de acuerdo con el perfil de las salas de hospitalización

\begin{tabular}{|l|c|c|c|c|c|}
\hline Perfil de las salas & $\begin{array}{c}\text { Pacientes } \\
\text { ingresados }\end{array}$ & \multicolumn{2}{|c|}{$\begin{array}{c}\text { Catéter } \\
\text { venoso } \\
\text { periférico }\end{array}$} & \multicolumn{2}{|c|}{ Flebitis } \\
\cline { 3 - 6 } & N. & RU & N. & $\%$ \\
\hline $\begin{array}{l}\text { Salas de cuidados } \\
\text { críticos }\end{array}$ & 392 & 177 & 0.45 & 10 & 5.6 \\
\hline Salas quirúrgicas & 532 & 185 & 0.35 & 6 & 3.2 \\
\hline Salas clínicas & 1223 & 725 & $0.59^{*}$ & 47 & 6.5 \\
\hline Total & 2282 & 1087 & 0.48 & 63 & 5.8 \\
\hline
\end{tabular}

$\mathrm{RU}$ : razón de uso. ${ }^{*} \mathrm{p}<0.05$.

elevada razón de uso de catéteres y de flebitis en salas seleccionadas.

Estudios previos describen la utilización de uno o más catéteres venosos periféricos en hasta el $80 \%$ de los pacientes hospitalizados ${ }^{1,2,7}$. La baja utilización en nuestro entorno podría estar relacionada con las características de la población de pacientes, especialmente la causa de ingreso y las indicaciones de acceso vascular para pacientes con condiciones clínicas poco complejas. El uso del acceso vascular debe explicarse en la mayoría de los pacientes debido al uso de antimicrobianos por vía intravenosa, que alcanza alrededor del $50 \%$ en una encuesta reciente realizada en la instalación (datos no publicados). Estudios previos publicados han demostrado que entre el 40 y el 50\% admitidos en esta instalación utilizan antimicrobianos ${ }^{8}$. Estudios publicados recientemente describen la prevalencia de flebitis en el 15.4, 31.8 y $36 \% 5,6$. De manera similar, los estudios habían encontrado una mayor prevalencia de flebitis en el Departamento médico (medicina interna, geriatría, cardiología) que en el Departamento quirúrgico ${ }^{5,9}$. La baja prevalencia de flebitis podría explicarse por el comportamiento del uso de CVP en el hospital u otros factores que requirieron estudios adicionales. Entre otros, podría ser importante evaluar la calidad del proceso de la pesquisa de flebitis, 
la justificación de los ingresos de los pacientes y el cumplimiento de las prácticas de prevención durante la inserción y el mantenimiento de catéteres.

Es importante tener en cuenta que las prácticas básicas de inserción y mantenimiento implementadas en países de bajos recursos podrían ser un riesgo para aumentar la prevalencia de flebitis y otros efectos adversos relacionados con el acceso vascular, incluidas las infecciones del torrente sanguíneo.

Se deben considerar hallazgos importantes para futuras acciones de mejora. El $80 \%$ de las flebitis se identificó en cinco salas de hospitalización (cuatro clínicas y una crítica), y se observó una mayor tasa de utilización en los departamentos clínicos. En estos servicios deben dirigirse, con prioridad, las acciones de mejora de la calidad.

Este estudio tiene varias limitaciones. Solo considera la evidencia de flebitis durante la encuesta, y este efecto adverso podría desarrollarse después de 48 horas de extracción del catéter. Esto podría subestimar la prevalencia de flebitis. Además, el objetivo del estudio no incluyó otros problemas relacionados con la indicación, el uso y el cumplimiento de las prácticas de cuidados de los CVP relacionados con la prevalencia de flebitis. La fortaleza de la pesquisa es focalizar las áreas problema susceptibles de ser modificadas con intervenciones específicas.

\section{Conclusión}

Este estudio proporciona información sobre el uso de catéteres periféricos y flebitis que debe utilizar el programa local de mejora de la calidad. Se requieren investigaciones adicionales para identificar la calidad de las indicaciones del catéter, la inserción, el mantenimiento y otras cuestiones relacionadas con el riesgo de flebitis.

\section{Agradecimientos}

Los autores desean agradecer el apoyo de la Sra. Guillermina Fernández Sánchez durante la investigación.

\section{Conflicto de intereses}

Los autores declaran no tener conflicto de intereses alguno.

\section{Financiamiento}

Los autores no recibieron patrocinio para llevar a cabo este artículo.

\section{Responsabilidades éticas}

Protección de personas y animales. Los autores declaran que para esta investigación no se han realizado experimentos en seres humanos ni en animales.

Confidencialidad de los datos. Los autores declaran que en este artículo no aparecen datos de pacientes.

Derecho a la privacidad y consentimiento informado. Los autores declaran que en este artículo no aparecen datos de pacientes.

\section{Bibliografía}

1. Capdevilla Renlu A, Capdevilla JA. Peripheral venous catheter: a dangerous weapon. Key points to improve its use. Rev Clin Esp. 2017;217(8):464-7.

2. Mattox EA. Complications of Peripheral Venous Access Devices: Prevention, Detection, and Recovery Strategies. Critical Care Nurse. 2017;37(2):e1-e14.

3. Zhang L, Cao S, Marsh N, Ray-Barruel G, Flynn J, Larsen E, et al. Infection risks associated with peripheral vascular catheters. J Infect Preven. 2016;17(5):207-13.

4. Uslusoy $\mathrm{E}$, Mete $\mathrm{S}$. Predisposing factors to phlebitis in patients with peripheral intravenous catheters: A descriptive study. J Am Acad Nurse Pract. 2008;20:172-80.

5. Atay S, Sen S, Çukurlu D. Phlebitis-related Peripheral Venous Catheterization and the Associated Risk Factors. Niger J Clin Pract. 2018; 21:827-31.

6. Cicolini G, Manzoli L, Simonetti V, Flacco ME, Comparcini D, Capasso L, et al. Phlebitis risk varies by peripheral venous catheter site and increases after 96 hours: a large multi-center prospective study. J Adv Nur. 2014;70(11):2539-49.

7. Hadaway L. Short peripheral intravenous catheters and infections. J Infus Nursing. 2012;35(4):230-40.

8. Guanche Garcell H, Pisonero Socias JJ, Enseñat Sánchez R, Fiterre Lancis I, Mir Narbona I, García Arzola B, et al. Impacto de un programa de control de la calidad de la prescripción de antibióticos en un hospital de La Habana, Cuba. Rev Panam Salud Publica. 2011;30(6):598-602.

9. Mestre Roca G, Berbel Bertolo C, Tortajada Lopez P, Gallemi Samaranch G, Aguilar Ramirez MC, Cayla Buqueras J, et al. Assessing the influence of risk factors on rates and dynamics of peripheral vein phlebitis: an observational cohort study. Medicina Clínica. 2012;5:185-91. 